\title{
The mnemonic effects of recall on immediate retention
}

\author{
James S. NaIrne ANd David A. Ceo \\ Purdue University, West Lafayette, Indiana \\ AND \\ Matthew B. Reysen \\ University of Mississippi, Oxford, Mississippi
}

\begin{abstract}
In three experiments, we investigated the mnemonic effects of an initial recall on later recall in an immediate memory setting. Recall is generally assumed to interfere with the recall of subsequent items (output interference), but previous experiments have failed to control for the confounding effects of time. In the experiments reported here, the passage of time was held constant on all trials; what varied was whether an additional item was recalled (or simply presented) during the retention interval. The results revealed clear evidence of recall's mnemonic effects, but output interference seemed strongest when the initial recall was of an item that followed the target item in the memory list. When participants initially recalled an item immediately preceding the target, target recall improved. This pattern of results places constraints on current models of immediate retention.
\end{abstract}

The act of recall can be either self-propagating or selflimiting (Roediger, 1978). In free recall, recalling one item often facilitates the recall of another, particularly if those items are related along a conceptual or temporal dimension (Deese, 1959; Kahana, 1996). Recalled items potentially serve as cues for retrieving additional items; this, in turn, enables the recall process to self-propagate (see Raaijmakers \& Shiffrin, 1981). Yet recall can be an important source of forgetting as well. The recall process takes time, which increases the effective retention interval for unrecalled items, and unrecalled items can be actively inhibited as a consequence of earlier retrievals (see Anderson, 2003; Bjork, 1989).

The self-limiting qualities of recall are particularly apparent in immediate serial recall, a task that requires events to be recalled in sequence. One of the signature characteristics of immediate serial recall is primacy: Beginning items are recalled best, even though early list items are susceptible to interference from later items during presentation (see Murdock, 1968). Various accounts of the primacy gradient have been proposed (e.g., early items receive a richer or stronger encoding; Farrell \& Lewandowsky, 2002; Page \& Norris, 1998), but the interference arising directly from output-so-called output interference-is widely assumed to play a significant role (see Nairne, 1990; Oberauer, 2003).

Evidence for output interference in immediate retention comes from several sources: First, when only a single item is tested on a given trial, either through probed recall or recognition, one typically finds large recency effects and substantially reduced primacy (Sternberg, 1969; Waugh \& Norman, 1965). Similar patterns are found for backward recall: Items recalled early, in this case the recency items, are recalled best, and few or no primacy effects are often found (Madigan, 1971; Thomas, Milner, \& Haberlandt, 2003). Finally, when list items are tested in random order after list presentation, or when participants are cued to begin recall at various points in the list, performance generally declines as a function of the item's position in the testing sequence (Cowan, Saults, Elliott, \& Moreno, 2002; Oberauer, 2003; Tulving \& Arbuckle, 1963).

Defined in terms of testing position, the evidence for output interference is clear and unequivocal. However, in each of the relevant experiments conducted to date, testing position has been confounded with the passage of time. Items tested (or output) late in a sequence, by definition, suffer from a longer effective retention interval than those tested (or output) early. Although time per se may not control performance in most immediate recall settings (Lewandowsky, Duncan, \& Brown, 2004; Nairne, 2002), events occurring in time can certainly play a significant role. To attribute output interference to the recall process itself, as opposed to decay or some other form of interpolated interference, requires an unconfounding of time and output position (Cowan et al., 2002; Oberauer, 2003; Roediger, 1978). The present experiments were designed, in part, to resolve this confounding.

To develop an adequate theoretical account of immediate retention, the mnemonic effects of the recall process need to be understood. Output interference is widely con- 
sidered to be an important factor in immediate recall but, surprisingly, it has received scant attention in formal models (see Farrell \& Lewandowsky, 2004). Typically, the negative effects of recall are attributed solely to the passage of time (Brown, Neath, \& Chater, 2002; Henson, 1998; Page $\&$ Norris, 1998) or, if implemented as interference, recall is assumed to add random "noise" to the representations of all nonrecalled items (Brown, Preece, \& Hulme, 2000; Farrell \& Lewandowsky, 2004). No distinctions are drawn between the recall of a particular list item (say, item $n$ ) and any other form of distracting activity (e.g., counting backward by threes) — both simply lead to a general degradation of existing memory traces.

Yet, is it reasonable to assume that the mnemonic effects of recall are nonspecific? As noted above, in addition to its self-limiting characteristics, recall has self-propagating properties. Kahana and Caplan (2002) have shown that recall of item $n$ in a serial list is better if it is cued with the immediately preceding item $(n-1)$ than with the immediately following item $(n+1)$. Serra and Nairne (2000) found that participants were better able to reconstruct the serial positions of some list items if other list items were placed in their appropriate presentation positions at test. Thus, rather than producing interference, the recall of item $n-1$ might enhance the recall of item $n$, and other nearby neighbors, and only impair the recovery of items occurring at more temporally distant positions in the list. Such a finding, if true, would place important constraints on existing models of immediate recall.

The present experiments were designed to achieve precise control over both temporal delay and output order. Participants received short lists of six items, followed by a probe that prompted recall of an item from a particular serial position in the list (e.g., $3=$ ?, where " 3 " refers to the third item in the just-presented list). The delay between the target probe and the end of the list was fixed on every trial (also see Ellis \& Anders, 1969). Of main interest was the activity that occurred during the fixed retention interval. In Experiment 1, on a random half of the trials, the retention interval was interrupted by recall of an additional list item. Experiment 2 employed a similar design, but controlled for the effect that an initial recall might have on response set size. Finally, in Experiment 3, participants were asked to either recall or copy a list item prior to final target recall. In each experiment, the passage of time was equated between conditions, thus allowing us to determine what effect, if any, an initial recall might have on later target recall.

\section{EXPERIMENT 1}

In Experiment 1, participants received 60 lists, each containing six unique items. Items were displayed visually, and the final list item was followed by a filled (double recall) or unfilled (single recall) retention interval. Regardless of trial type, the retention interval lasted $8 \mathrm{sec}$ and always ended with the appearance of a position prompt of the form $X=$ ?, where $X$ varied randomly from
1 to 6 , to represent the 6 serial positions of the list. All serial positions were tested equally often in both the single and double recall conditions.

On double recall trials, participants were required to recall two list items, one immediately following list presentation and the other at the end of the 8-sec retention interval. Because we were interested in the effect of an initial recall on a final target recall, an extra cue was given to facilitate the first recall; the prompt was of the form $X=$ a__?, where the extra cue was the first letter of the item occupying the tested position. The same item was never tested twice (i.e., as both the initial and final recall), and all possible combinations of initial and target recalls were sampled across trials (e.g., $n-1$ and $n ; n+1$ and $n, n-2$ and $n$, and so forth). This design enabled us to examine the effect of an initial recall on final target recall, but also to investigate whether the serial relationship between the two tested items mattered.

\section{Method}

Participants and Apparatus. Participants were 55 Purdue University undergraduates who participated for credit in an introductory psychology course. Everyone was tested individually in a session lasting approximately $35 \mathrm{~min}$. Stimuli were presented and controlled by IBM-compatible computers.

Design and Materials. Sixty 6-item memory lists were presented in a 6 (serial position) $\times 2$ (single vs. double recall) within-subjects design. List items were medium-frequency, lowercase words drawn from the Paivio, Yuille, and Madigan (1968) norms. No word was repeated within the experiment; words within each list always began with a different first letter.

Procedure. Each trial began with a ready prompt ("Here is the Study List") that remained centered on the screen for 2,000 msec. Next, list items appeared sequentially, for 2,000 msec each, also centered on the screen. There were two trial types: single recall and double recall. On the 30 single recall trials, list presentation was followed immediately by an unfilled retention interval lasting $8,000 \mathrm{msec}$; the words "Please Wait a Moment" appeared on the screen for $7,500 \mathrm{msec}$, followed by a $500-\mathrm{msec}$ blank interval. The retention interval ended with a position prompt $(X=$ ?, where $X$ was a digit from 1 through 6$)$; the participants were asked to recall the item that had appeared at the queried serial position in the list. A text box appeared just below the recall prompt and the participants were allowed 7,500 msec to type in the appropriate recall response; $500 \mathrm{msec}$ later, a new trial began. Each serial position was tested equally often and randomly throughout the session.

On double recall trials, an initial recall prompt appeared (e.g., $X=\mathrm{a} \quad$ ) immediately following presentation of the list. The participants were allowed $7,500 \mathrm{msec}$ to enter a recall response. As noted above, the first letter of the tested item was given to the participants to aid recall. A blank screen lasting $500 \mathrm{msec}$ preceded the appearance of the second recall prompt $(X=$ ?), which matched the prompt on single recall trials in all respects. Across the 30 double recall trials, all possible combinations of serial positions were tested equally often in random order. Single and double recall trials occurred randomly throughout the experimental session. Two practice lists, one of each type, preceded the experimental trials.

\section{Results and Discussion}

The data of main interest are shown in Figure 1. The proportion of correct target recall is plotted as a function of serial position for both the single and double recall conditions. The data for the first recall in the double recall 


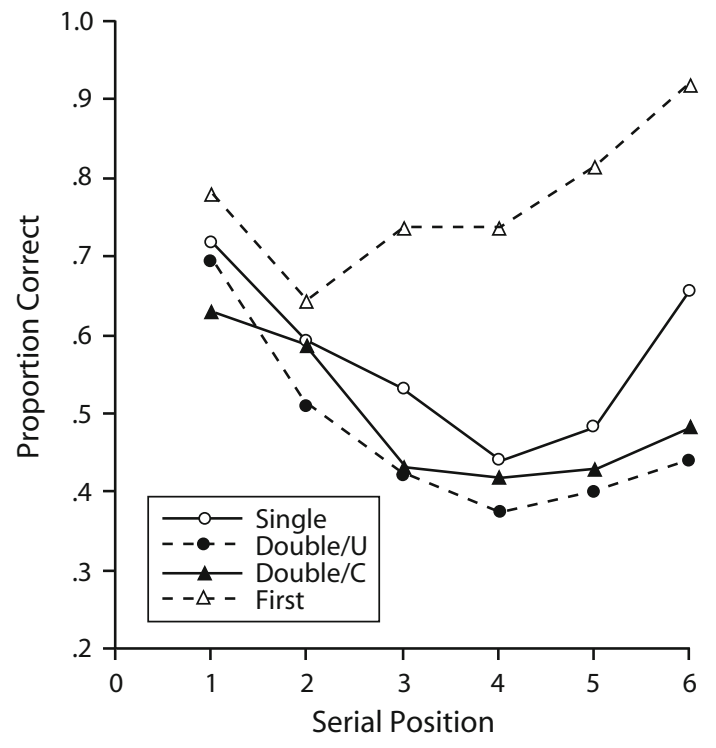

Figure 1. The mean proportion of correct target recall for the single and double recall trials in Experiment 1. First recall data on double recall trials are also shown.

condition are also shown, as well as final target recall conditionalized on a correct initial recall. The mean proportion correct for the first recall was .77.

An initial ANOVA was conducted, comparing target recall in just the single and double (unconditionalized) conditions. The analysis revealed a significant effect of trial type $\left[F(1,54)=38.93, M S_{\mathrm{e}}=.040\right]$, serial position $\left[F(5,270)=24.73, M S_{\mathrm{e}}=.051\right]$, and the trial $\times$ position interaction $\left[F(5,270)=2.84, M S_{\mathrm{e}}=.041\right]$. Somewhat surprisingly, the serial position curves showed marked primacy in both conditions, which differs from the recencydriven curves that are more typical of probed recall (e.g., Ellis \& Anders, 1969; Murdock, 1968; Waugh \& Norman, 1965). Any effect of output interference should have been sharply reduced in these conditions, so the presence of significant primacy is noteworthy. In the single recall condition, in particular, only a single target recall was required on a given trial. We will return to this issue later in the discussion.

Most importantly, the data revealed clear differences in final target recall between the single and double recall conditions. With the exception of the first serial position, the participants performed consistently better on the single recall trials. Because the retention interval was equated across conditions in this experiment, this decrement on double recall trials can be attributed to the initial recall rather than to the passage of time per se. Moreover, because the participants could not predict trial type during presentation of the list, we can assume that list items were comparably encoded across the two trial conditions. A similar pattern of results was found when target recall in the double recall condition was conditionalized on correct initial recall. The average decrement (single vs. double) dropped from .10 to .08 , but the ANOVA mirrored the pattern found for the unconditionalized data. ${ }^{1}$
Of course, the fact that final target recall is lower in the double recall condition tells us little about the processes responsible for the impairment. It is possible, for example, that any interruption during a retention interval hurts performance, either because the activity interferes with residual list memory traces (e.g., features are overwritten; see Nairne, 1990) or because the interruption disrupts normal rehearsal strategies. In fact, initial recall might serve merely as a kind of distractor task, much like counting backward or articulatory suppression. As noted earlier, when output interference is modeled formally, it is often implemented as a general overall effect (i.e., noise added to the memory traces of all unrecalled items; Farrell \& Lewandowsky, 2004).

To explore this possibility, we next examined the content relationship between first and second recalls. If an initial recall simply interrupts processing (e.g., rehearsal), or adds general noise, then first recall content should be irrelevant; that is, it should not matter whether the participant is prompted to recall item $n-1$, or any other list item, prior to the recall of item $n$. Figure 2 shows final target recall in the double recall condition as a function of whether the first recall was a nearby neighbor of the target word during list presentation. The columns marked $n-1$ and $n-2$ show average target recall when participants initially recalled an item immediately preceding the target $(n-1)$ or two back $(n-2)$; the remaining columns show target recall when the participants initially recalled items that followed the target in the list. To avoid confounding distance and serial position, we restricted this analysis to targets from the middle two serial positions in the list (Positions 3 and 4). In this way, distance (one back vs. two back) and direction (preceding vs. following) of the first recall could be examined for targets occurring at exactly the same serial positions.

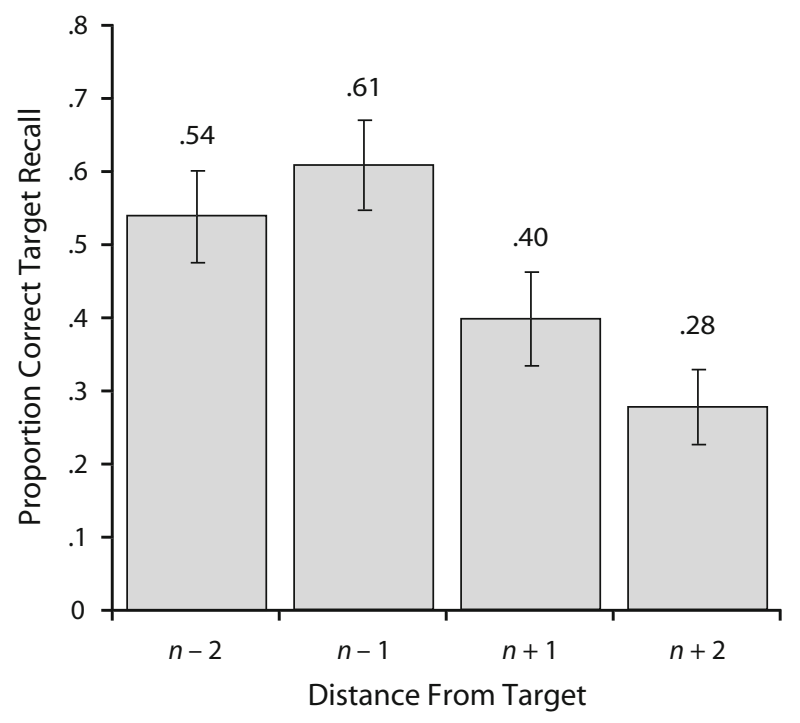

Figure 2. The mean proportion of correct target recall on double recall trials in Experiment 1, plotted as a function of the distance of the first recall from the target. Data are conditionalized on a correct initial recall. 
Figure 2 shows quite clearly that first recall content importantly influenced final target recall performance. An overall ANOVA on these data, which were conditionalized on correct initial recall, revealed a significant effect of direction: Final target recall was significantly better when participants initially recalled an item preceding the target item in the list $\left[F(1,44)=14.77, M S_{\mathrm{e}}=.167\right]$. A somewhat similar pattern of asymmetry was reported by Kahana and Caplan (2002), who found that forward cuing, with items immediately preceding target items in a serial list, led to better performance than backward cuing. In the present case, of course, participants were not formally cued; instead, recalling a preceding item benefited target performance more than recalling an item that followed the target in the list. There is some suggestion of a distance gradient as well-that is, better recall if the initial recall was of a nearby item (e.g., $n-1$ vs. $n-2)$ - but distance was only marginally significant in the ANOVA $[F(1,44)=$ $\left.3.57, M S_{\mathrm{e}}=.126, p<.07\right]$; the interaction of direction and distance failed to reach significance $[F(1,44)<1]$.

Figure 3 provides a further breakdown of the content recall data by serial position and shows performance relative to the single recall condition; again, the data were conditionalized on a correct initial recall. Here, we were interested in whether the negative effect of output interference might be confined to the recall of items that followed target items in the list. (For the sake of generality, we present the data here for all relevant serial positions rather than focusing exclusively on Positions 3 and 4.) There is variability in this subset of the data because of the reduced number of observations, but the interfering effects of initial recall do seem to be restricted primarily to "backward" initial recalls. For the interior serial positions, in particular, a "forward" initial recall $(n-1, n-2)$ appeared to facilitate rather than impair target recall; there is certainly little evidence for a general impairment. For

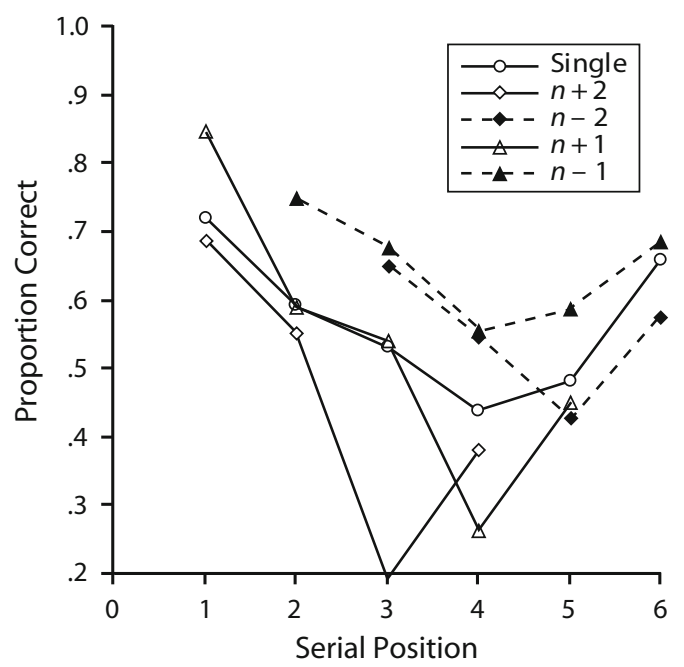

Figure 3. The mean proportion of correct target recall in Experiment 1 for trials in which the initial recall either preceded $(n-1, n-2)$ or followed $(n+1, n+2)$ the target in the list. Data from the single trials are shown for comparison purposes. backward initial recalls $(n+1, n+2)$, performance in the double recall conditions was consistently below single recall performance. Given that normal serial recall always occurs in the forward direction, these data suggest that the act of recall may have only minimal negative effects on subsequent recall performance.

Finally, as noted earlier, the serial position curves for target recall in both the single and double recall conditions show considerable primacy. At first glance, this is perplexing because probed recall tests of the type employed here typically produce serial position curves with strong recency gradients (e.g., Murdock, 1968). However, importantly, target recall in the present experiment did not occur immediately following list presentation; instead, it occurred after a delay of $8 \mathrm{sec}$. Examination of the serial position curve for the first recall, which occurred immediately after completion of list presentation, reveals the more typical patternreduced primacy and a strong recency gradient. The fact that recency items are particularly hurt by a lengthening of the retention interval is not unusual in studies of shortterm retention (Bjork, 1999; Ellis \& Anders, 1969; Neath \& Knoedler, 1994; Wright, Santiago, Sands, Kendrick, \& Cook, 1985). This kind of time-dependent recency-toprimacy shift has been attributed by some to changes in the temporal discriminability of items over time (see Knoedler, Hellwig, \& Neath, 1999, for a full discussion).

\section{EXPERIMENT 2}

Experiment 2 was designed primarily to replicate Experiment 1, with an additional control condition. Although Experiment 1 provided clear evidence for output interference, at least when participants initially recalled items that followed the target item in the list, the effects were not particularly large (at least in comparison with the extent of the primacy gradient). It is conceivable, though, that target recall in the double recall condition might have been enhanced somewhat because of a reduction in response set size at the point of test. Response set size refers to the number of possible responses that are available for recall. Response set size diminishes as recall proceeds because recalled items are eliminated from the pool of possible recall candidates (see Cowan et al., 2002). In the double recall condition, given a correct initial recall, participants faced a candidate pool of only five items at the point of test, as opposed to six in the single recall condition. This reduction in response set size might have conferred an advantage to the double recall condition leading to an underestimation of measured output interference.

To control for differences in response set size, on each trial (single and double) all of the list items were re-presented at test except for the target prompted by the recall cue. Participants were told that the five re-presented items could be used to help them recall the prompted target item. In all other respects, Experiment 2 was a direct replication of Experiment 1.

\section{Method}

Participants and Apparatus. Participants were 64 Purdue University undergraduates who participated for credit in an introductory 
psychology course. Everyone was tested individually in a session lasting approximately $35 \mathrm{~min}$. Stimuli were presented and controlled by IBM-compatible computers.

Design and Materials. As in Experiment 1, the participants received 60 memory lists in a 6 (serial position) $\times 2$ (single vs. double recall) within-subjects design. The materials, list construction, timing intervals, and design matched Experiment 1 in all respects except for the test display. Immediately below the target recall prompt $(X=$ ?) and the response box, the five untested list items reappeared in their original order of presentation. The items were displayed in a line, and an $X$ appeared in the spot occupied by the to-be-recalled item. On double recall trials, the initial recall followed exactly the same format as in Experiment 1.

Procedure. All aspects of the procedure from Experiment 1 were matched in Experiment 2 except that participants were told to expect nontested list items concurrent with the target recall prompt. It was suggested that these items might help them recall the prompted target.

\section{Results and Discussion}

Figure 4 shows target recall from the single and double recall conditions, as well as the first recall data. The mean proportion of correct first recall was .76. Overall, the data replicate the general patterns of Experiment 1 in virtually all respects. An ANOVA comparing target recall in the single and double (unconditionalized) conditions revealed a significant effect of trial type $\left[F(1,63)=27.03, M S_{\mathrm{e}}=\right.$ $.056]$ and serial position $\left[F(5,315)=27.45, M S_{\mathrm{e}}=.047\right]$; the trial type $\times$ serial position interaction was significant as well $\left[F(5,315)=4.07, M S_{\mathrm{e}}=.051\right]$. As in the prior experiment, the target recall curves show a strong primacy gradient, target recall is clearly impaired in the double recall condition, and the impairment increased in size in the recency portion of the list.

However, there is no evidence that providing list items at test increased the size of the impairment. In Experiment 1 , collapsing across serial position, the difference

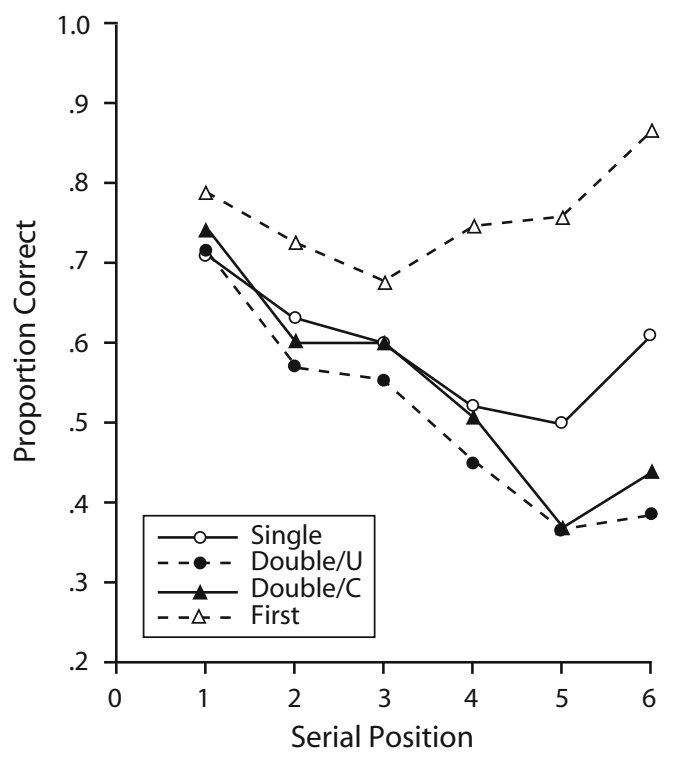

Figure 4. The mean proportion of correct target recall for the single and double recall trials in Experiment 2. First recall data on double recall trials are also shown.

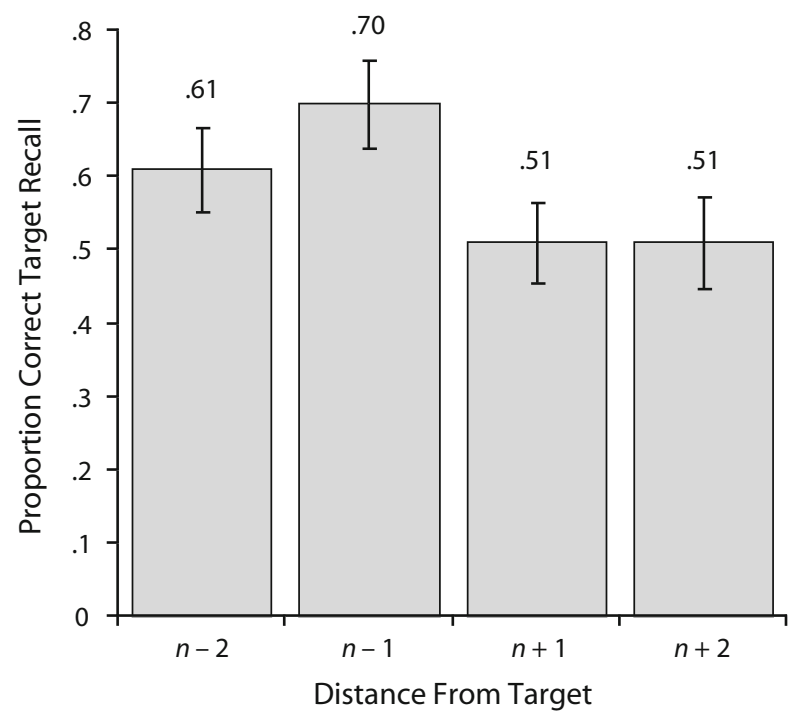

Figure 5. The mean proportion of correct target recall on double recall trials in Experiment 2, plotted as a function of the distance of the first recall from the target. Data are conditionalized on a correct initial recall.

between the single and double recall conditions averaged .10; in Experiment 2, the average was .09. Furthermore, when target recall in the double recall condition is conditionalized on a correct first recall, the average drops to .05 and output interference is evident only for the last two serial positions. An ANOVA comparing target recall in the single recall and conditionalized double recall conditions also revealed a significant effect of trial type $[F(1,62)=$ $\left.6.81, M S_{\mathrm{e}}=.070\right]$, serial position $[F(5,310)=20.98$, $\left.M S_{\mathrm{e}}=.062\right]$, and the trial type $\times$ serial position interaction $\left[F(5,310)=3.20, M S_{\mathrm{e}}=.065\right]$. Overall, though, the magnitude of output interference pales in comparison with the size of the primacy effect, in which performance drops roughly in half, from the first to fifth serial positions.

Figure 5 shows how the temporal (or serial) relationship between the first and second recall affected performance in Experiment 2. Final target recall is shown as a function of whether the first recall was a nearby neighbor of the target word during list presentation; once again, the analysis was restricted to targets from the middle two positions in the list. Replicating Experiment 1, an ANOVA revealed a highly significant effect of direction $[F(1,50)=6.52$, $\left.M S_{\mathrm{e}}=.158\right]$; neither the effect of distance nor the interaction approached significance in the analysis. Initially recalling an item that preceded the target item in the list helped performance more than recalling an item that followed the target item in the list.

Figure 6 shows how these content-based recall effects compare with the single recall condition. Because of the reduced number of observations per point-for example, each participant received only one $n-1$ trial at the second serial position-there is considerable variability in the data; however, the general trends replicate the patterns seen in Experiment 1. There is little impairment for forward initial recalls $(n-1, n-2)$ except in the recency por- 


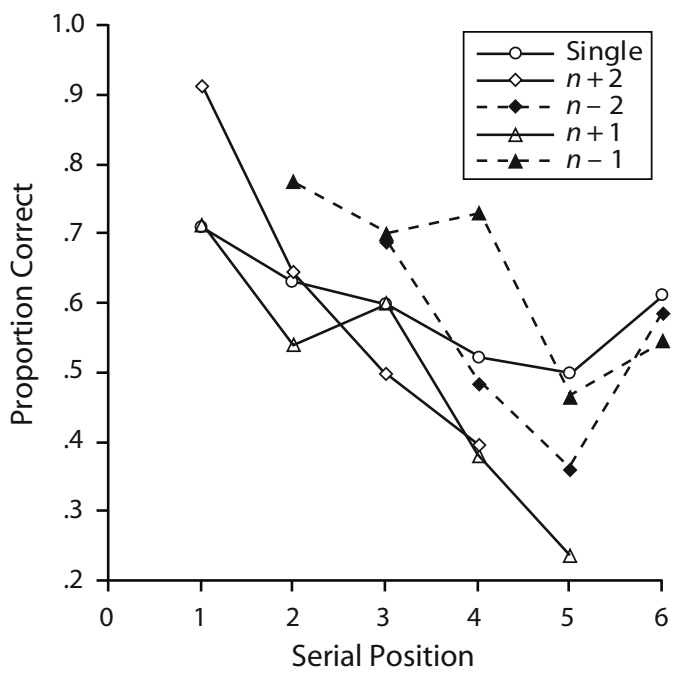

Figure 6. The mean proportion of correct target recall in Experiment 2 for trials in which the initial recall either preceded $(n-1, n-2)$ or followed $(n+1, n+2)$ the target in the list. Data from the single trials are shown for comparison purposes.

tion of the list; in fact, there is evidence for facilitation across the interior serial positions. For backward initial recalls $(n+1, n+2)$, performance generally drops below single recall. In order to provide some statistical evidence for these trends, we collapsed across Experiments 1 and 2 and examined performance only for the nearest neighbors $(n+1, n-1)$. Averaging across serial positions $2-5$, target recall on $n-1$ trials was significantly better than performance on single recall trials [.64 vs. .54; $t(94)=2.75$ ]; in contrast, target recall on $n+1$ trials was significantly lower than performance on single recall trials [.45 vs. .54; $t(94)=-2.71]$.

Finally, the recency-primacy shift found between initial recall and target recall in the single recall condition reappeared in Experiment 2. Immediate testing of a single list item led to a serial position curve marked by a strong recency gradient; probed recall of a single list item after a delay (in this case, $8 \mathrm{sec}$ ) led to a strong primacy gradient. We consider the theoretical implications of this shift in more detail in the General Discussion.

\section{EXPERIMENT 3}

In both of the previous experiments, interrupting a retention interval with a request to recall one of the list items had significant mnemonic effects on later recall of a second list item (relative to a single recall condition). Whether the effect was positive or negative depended on the content of the first recall-specifically, its temporal relationship to the target item. However, we still cannot be certain that the locus of these mnemonic effects truly lies in a generative recall process; instead, the effects might arise simply because one of the list items is repeated, by virtue of its recall, during the retention interval. Strengthening some list items at the expense of others can produce interference (e.g., the list strength effect, Ratcliff, Clark, \& Shiffrin, 1990), as can the presentation of part-list cues at the point of test (Nickerson, 1984). The point of Experiment 3 was to compare recall with simple presentation of a list item during the retention interval.

The design of Experiment 3 matched the previous experiments in all respects except that the participants were required to either recall or simply copy one of the list items during the retention interval. On half of the trials, as in Experiments 1 and 2, the retention interval began with the appearance of a recall prompt $\left(X=\mathrm{a} \_\right.$_ $)$; on the remaining trials, a similar prompt appeared but the entire word was displayed ( $X=$ apple). For these trials, the participants were told simply to copy the presented word into the response box.

\section{Method}

Participants and Apparatus. The participants were 44 Purdue University undergraduates who participated for course credit. Everyone was tested individually in a session lasting approximately $35 \mathrm{~min}$.

Design and Materials. The design and materials matched those used in Experiments 1 and 2. However, single recall trials from the previous experiments were changed into "copy" trials. Copy trials matched double recall trials in all respects except for the probe $(X=$ a__ vs. $X=$ apple). For both double recall and copy trials, all possible combinations of serial positions were tested as the initial and final prompts.

Procedure. The procedural details matched Experiment 1 except that instructions were given to retype the probe word on copy trials.

\section{Results and Discussion}

In Experiment 3, everything remained the same on all trials, including the interruptive properties of the initial prompt, except for the requirement to generate (or not) an initial item. Figure 7 shows the data from the copy trials and double recall trials (conditionalized and unconditionalized), plotted as a function of serial position. A strong primacy gradient is evident for all conditions, but there is no main effect of trial type. An ANOVA comparing the copy and unconditionalized double recall trials revealed only a significant effect of serial position $\left[F(5,215)=16.38, M S_{\mathrm{e}}=\right.$ $.065]$. First recall data are also shown, plotted as a function of serial position (the mean proportion correct was .87).

The null effect of trial type suggests that it is probably the cuing properties of initial recall that are mediating the later mnemonic effects on target recall. This is an important finding because some theoretical accounts of output interference, such as those appealing to retrieval-induced inhibition (Anderson, 2003), draw a critical distinction between recall and mere presentation. Inhibition is assumed to occur only as a consequence of selective retrieval: We suppress competing memories in an effort to override their potential interfering effects. Merely presenting list items for additional study should not initiate selective retrieval and therefore should produce no inhibitory effects (Anderson, 2003). Given that participants performed similarly on both trial types, retrieval-induced forgetting may play little, if any, role in remembering lists of items over the short term. ${ }^{2}$

Further insight is provided by analyzing the content relationship between the initial presented/recalled item and 


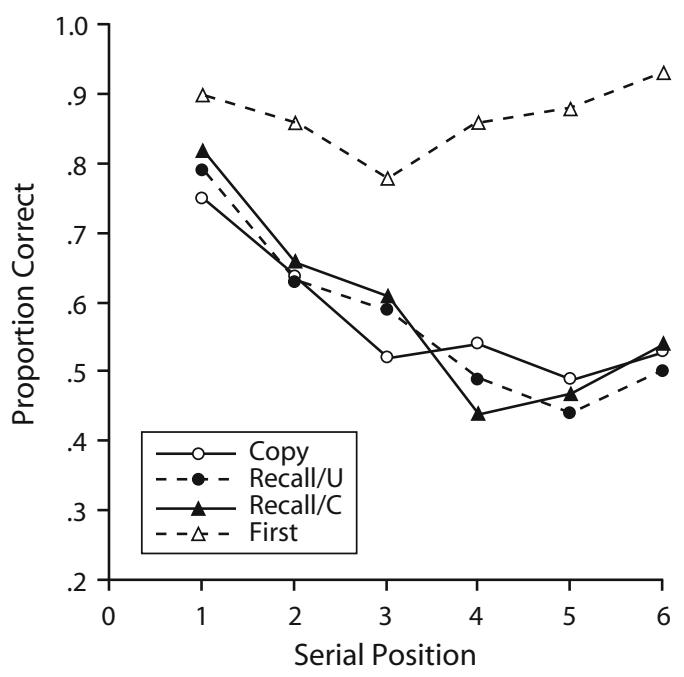

Figure 7. The mean proportion of correct target recall for the double recall trials and "copy" trials in Experiment 3.

final target recall. Table 1 presents the data for both the copy and the double recall trials. Because conditionalizing required us to eliminate participants who failed to provide observations for each of the relevant conditions, we performed separate ANOVAs for each trial type. However, as the data show, similar patterns were found for each condition. Turning first to the copy trials, the ANOVA revealed a significant effect of direction: Final target recall was significantly better when participants were presented with an item preceding the target item in the list $[F(1,43)=$ $\left.13.72, M S_{\mathrm{e}}=.087\right]$; neither distance $[F(1,43)=2.79$, $\left.M S_{\mathrm{e}}=.086\right]$ nor the interaction $\left[F(1,43)=1.73, M S_{\mathrm{e}}=\right.$ .099 ] was significant in the analysis. A similar pattern was found for double recall trials, although the main effect of direction was only marginally significant $[F(1,29)=3.54$, $\left.M S_{\mathrm{e}}=.133, p<.07\right]$. As in the previous experiments, there was some suggestion of a distance effect (e.g., $n-1$ vs. $n-2)$, but it did not reach conventional levels of significance $\left[F(1,29)=2.60, M S_{\mathrm{e}}=.097\right] .^{3}$

The content recall data reinforce the idea that it is not recall per se that is influencing final target recall, but the occurrence of a particular list item during the retention interval. Interpolated items presumably have cuing properties and, depending on their temporal relationship to the final target, can facilitate or impair later recall. The fact that the temporal (or positional) relationship between items mattered demonstrates that it is not merely the interruptive properties of the interpolated event that influence final target recall. If an initial recall, or presentation, simply stops or delays selective rehearsal, leading to impaired target recall relative to a blank retention interval, then we would expect the impairment to be general and nonspecific, which it clearly is not.

\section{GENERAL DISCUSSION}

The present experiments were designed to provide a preliminary examination of the mnemonic effects of recall on immediate retention. Although the recall of one item is generally assumed to interfere with the recall of subsequent items (output interference), previous experiments have failed to control for the confounding effects of time. In the experiments reported here, the passage of time was held constant on all trials; what varied was whether an additional item recall (or presentation) occurred during the retention interval.

All three experiments revealed that an initial recall had clear mnemonic effects on later target recall. In Experiments 1 and 2, performance on double recall trials was consistently below single recall performance, demonstrating the presence of output interference. However, the size of the impairment depended on the serial positions of the two tested items. Target recall performance was significantly better when participants initially recalled an item that preceded the target item in the list. For some serial positions, in fact, recalling an item that immediately preceded the target actually facilitated performance relative to the single recall condition. These results suggest that the mnemonic effects of recall are not nonspecific, but rather depend on the temporal (or positional) relationship among the items in a list.

Output interference is often used as a vehicle for explaining memory phenomena, particularly the primacy effect in immediate serial recall (Cowan et al., 2002; Nairne, 1990; Oberauer, 2003). The act of recall is assumed to interfere with the memory traces of unrecalled items, either because those traces are overwritten or decay coincident with the passage of time. Current models of immediate retention acknowledge a role for output interference, but a nonspecific one: The act of recall takes time, which furthers decay (Page \& Norris, 1998), impairs discriminability (Brown et al., 2000), or adds noise to unrecalled memory traces; this, in turn, makes those traces harder to interpret (Farrell \& Lewandowsky, 2002). None of these models can, at present, handle both the facilitatory and inhibitory consequences of an initial recall without additional assumptions. Moreover, given that time was equated in our experiments, any simple time-based mechanism, such as decay, will not be sufficient to explain the mnemonic effects that the recall of one item has on the recall of another.

The fact that a "forward" initial recall had a generally positive effect on target recall suggests that early list items may act as effective retrieval cues for later recalls. As noted earlier, Kahana and Caplan (2002) showed that recall of item $n$ in a serial list is better if it is cued with the immediately preceding item $(n-1)$ than with the immediately following item $(n+1)$. Such data seem consistent with traditional chaining models: People may form associations among list items that are stronger, or easier to access, in the forward direction (see also Serra \& Nairne,

Table 1

Target Recall As a Function of Position of First Recall

\begin{tabular}{lcccc}
\hline & \multicolumn{4}{c}{ Distance From Target } \\
\cline { 2 - 5 } \multicolumn{1}{c}{ Condition } & $n-2$ & $n-1$ & $n+1$ & $n+2$ \\
\hline Recall & .75 & .80 & .72 & .58 \\
Copy & .72 & .73 & .63 & .49 \\
\hline
\end{tabular}


2000). Associative asymmetries are not typically found in traditional paired-associate learning tasks (Kahana, 2002), which raises an additional puzzle for any general theory of memory. It is also certainly possible that position-based, or context-driven, models of serial recall can be tweaked to handle the facilitatory effects of item cuing. One might argue, for example, that access to the position code of item $n$ (or its associated context) makes it easier to access the position codes of nearby items, as long as those items precede the target item in time (see Kahana \& Caplan, 2002, for an extended discussion).

Output interference was documented most strongly when participants initially recalled items that followed the target item in the list-for example, recalling item $n+2$ prior to item $n$. There are several ways to interpret this result. One possibility is that recall always leads to the relative degradation of unrecalled traces (e.g., because of decay, interference, or the interruption of rehearsal), but these negative effects can be overcome in some instances by the positive effects of recall-based cuing. Given that cuing effects are strongest in the forward direction, output interference would be most noticeably "unmasked" for backward initial recalls. A second possibility is that initial backward recalls tend to disrupt participants' normal output strategies to a greater extent than forward recalls. If people are inclined to output (or rehearse) list items in sequence, as some evidence suggests (see Thomas et al., 2003), then requiring an out-of-sequence recall could be disruptive.

The results of Experiment 3 further suggest that it is the cuing properties of the interpolated event, rather than an act of recall per se, that should receive the main focus. Similar patterns of results were found when participants recalled or simply copied the interpolated event. Of course, in other contexts it has been suggested that the mere presentation of an item might induce a form of involuntary retrieval (see Crowder, 1976). In his analysis of part-list cuing, Roediger (1974) appealed to output interference as a mechanism to explain the reduction in recall of noncued list items. Presentation of list items as cues is analogous to recalling those same items, which, in turn, "pushes the attempted retrieval of other items farther out along a gradient of output interference" (Crowder, 1976, p. 347). Further experiments will be required to resolve this issue, although it seems certain that recall will turn out to have complex mnemonic effects.

The present experiments also suggest that output interference is unlikely to provide any kind of complete explanation for the primacy effect found in immediate serial recall. First, as just discussed, recall does not always lead to impairment, and its negative effects tend to occur primarily for backward initial recalls. Normal serial recall, of course, occurs in forward sequence. Second, considerable primacy effects were obtained here, even though the potential for output interference was minimized. On half of the trials, participants were required to recall only a single item, yet considerable primacy was found. It is conceivable that participants covertly rehearsed list items during the retention interval, and this covert activity produced interference, but it is difficult to understand why covert activity would yield interference when an actual overt recall produced interference only in some circumstances. Moreover, covert activity is likely to involve rehearsing items in sequence, and the present data suggest that forward recall produces little impairment.

At the same time, it is important to note that the present experiments controlled for the passage of time. In normal serial recall, recall is sequential and the accumulated effects of time may well contribute to the final shape of the serial position curve. Indeed, the present experiments provided evidence for a time-dependent recency-to-primacy shift. The serial position curve for initial recall, which occurred immediately following the list, was characterized primarily by recency; the strong primacy gradient appeared only when testing occurred after a delay. Again, one might appeal to the interfering effects of covert rehearsal to explain this shift, but there is little concrete evidence to support this account. Moreover, the recencyto-primacy shift has been well-documented in situations in which rehearsal is extremely unlikely to occur (e.g., Neath, 1993). The shift is better handled by temporal (or positional) distinctiveness accounts that appeal to how the discriminability of items changes with the passage of time (e.g., Brown et al., 2002).

Memory researchers have long recognized that recall can have both interfering and facilitatory effects. The cuing properties of recalled items help the recall process to self-propagate; the fact that recall unfolds over time increases the effective retention interval for unrecalled items and can be an important source of forgetting. The present experiments provide new evidence for each of these processes in an immediate memory context. Clearly, the act of immediate recall has mnemonic effects, and the nature of those effects depends importantly on the temporal (or positional) relationship between recalled and yetto-be-recalled items. Output interference, when it occurs, is neither nonspecific nor necessarily interfering; as such, the present data present a challenge for models and theoretical accounts of immediate retention.

\section{AUTHOR NOTE}

Correspondence concerning this article should be addressed to J. S. Nairne, Department of Psychological Sciences, Purdue University, 703 Third Street, West Lafayette, IN 47907-2081 (e-mail: nairne@ psych .purdue.edu).

\section{REFERENCES}

Anderson, M. C. (2003). Rethinking interference theory: Executive control and the mechanisms of forgetting. Journal of Memory \& Language, 49, 415-445.

BJORK, R. A. (1989). Retrieval inhibition as an adaptive mechanism in human memory. In H. L. Roediger III \& F. I. M. Craik (Eds.), Varieties of memory and consciousness: Essays in honour of Endel Tulving (pp. 309-330). Mahwah, NJ: Erlbaum.

BJORK, R. A. (1999). Recency and recovery in human memory. In H. L. Roediger III, J. S. Nairne, I. Neath, \& A. Surprenant (Eds.), The nature of remembering: Essays in honor of Robert G. Crowder (pp. 211232). Washington, DC: American Psychological Association.

Brown, G. D. A., Neath, I., \& Chater, N. (2002). A ratio model of scale-invariant memory and identification. Unpublished manuscript. 
Brown, G. D. A., Preece, T., \& Hulme, C. (2000). Oscillator-based memory for serial order. Psychological Review, 107, 127-181.

Cowan, N., Saults, J. S., Elliott, E. M., \& Moreno, M. V. (2002). Deconfounding serial recall. Journal of Memory \& Language, 46, 153-177.

CROWDER, R. G. (1976). Principles of learning and memory. Hillsdale, NJ: Erlbaum.

DEESE, J. (1959). Influence of inter-item associative strength upon immediate free recall. Psychological Reports, 5, 305-312.

Ellis, N. R., \& ANDERS, T. R. (1969). Effects of interpolated recall on short-term memory. Journal of Experimental Psychology, 79, 568-569.

FARRELl, S., \& LeWANDOWSKY, S. (2002). An endogenous distributed model of ordering in serial recall. Psychonomic Bulletin \& Review, 9, 59-79.

FARRELL, S., \& LEWANDOWSKY, S. (2004). Modelling transposition latencies: Constraints for theories of serial order memory. Journal of Memory \& Language, 51, 115-135.

Henson, R. N. A. (1998). Short-term memory for serial order: The start-end model. Cognitive Psychology, 36, 73-137.

Kahana, M. J. (1996). Associative retrieval processes in free recall. Memory \& Cognition, 24, 103-109.

KAHANA, M. J. (2002). Associative asymmetry and memory theory. Memory \& Cognition, 30, 823-840.

KAHANA, M. J., \& CAPLAN, J. B. (2002). Associative symmetry in probed recall of serial lists. Memory \& Cognition, 30, 841-849.

Knoedler, A. J., Hellwig, K. A., \& NeATH, I. (1999). The shift from recency to primacy with increasing delay. Journal of Experimental Psychology: Learning, Memory, \& Cognition, 25, 474-487.

LeWANDOWSKY, S., DunCAN, M., \& Brown, G. D. A. (2004). Time does not cause forgetting in short-term serial recall. Psychonomic Bulletin \& Review, 11, 771-790.

MADIGAN, S. A. (1971). Modality and recall order interactions in shortterm memory for serial order. Journal of Experimental Psychology, 87, 294-296.

MuRDOCK, B. B., JR. (1968). Serial order effects in short-term memory. Journal of Experimental Psychology Monographs, 76(4, Pt. 2), 1-15.

NaIRne, J. S. (1990). A feature model of immediate memory. Memory \& Cognition, 18, 251-269.

NAIRNE, J. S. (2002). Remembering over the short term: The case against the standard model. Annual Review of Psychology, 53, 53-81.

NeAth, I. (1993). Distinctiveness and serial position effects in recognition. Memory \& Cognition, 21, 689-698.

NEATH, I., \& KNOEDLER, A. J. (1994). Distinctiveness and serial position effects in recognition and sentence processing. Journal of Memory \& Language, 33, 776-795.

NICKERSON, R. S. (1984). Retrieval inhibition from part-set cuing: A persisting enigma in memory research. Memory \& Cognition, 12, 531-552.

OBERAUER, K. (2003). Understanding serial position curves in short-term recognition and recall. Journal of Memory \& Language, 49, 469-483.

PAGE, M. P. A., \& Norris, D. G. (1998). The primacy model: A new model of immediate serial recall. Psychological Review, 105, 761-781.

Paivio, A., Yuille, J. C., \& Madigan, S. A. (1968). Concreteness, imagery, and meaningfulness values for 925 nouns. Journal of Experimental Psychology Monographs, 76(1, Pt. 2).
RAAiJMAKers, J. G. W., \& Shiffrin, R. M. (1981). Search of associative memory. Psychological Review, 88, 93-134.

Ratcliff, R., Clark, S. E., \& SHIFFrin, R. M. (1990). The list-strength effect: 1. Data and discussion. Journal of Experimental Psychology: Learning, Memory, \& Cognition, 16, 163-178.

RoEDIGER, H. L., III (1974). Inhibiting effects of recall. Memory \& Cognition, 2, 261-269.

RoEDIGER, H. L., III (1978). Recall as a self-limiting process. Memory \& Cognition, 6, 54-63.

Serra, M., \& NaIrne, J. S. (2000). Part-set cuing of order information: Implications for associative theories of serial order memory. Memory \& Cognition, 28, 847-855.

STERNBERG, S. (1969). Memory scanning: Mental processes revealed by reaction time experiments. American Scientist, 57, 421-457.

Thomas, J. G., Milner, H. T., \& HaberlandT, K. F. (2003). Forward and backward recall: Different response time patterns, same retrieval order. Psychological Science, 14, 169-174.

Tulving, E., \& ARBUCKLE, T. Y. (1963). Sources of intratrial interference in immediate recall of paired associations. Journal of Verbal Learning \& Verbal Behavior, 1, 321-334.

Waugh, N. C., \& Norman, D. A. (1965). Primary memory. Psychological Review, 72, 89-104.

WRight, A. A., Santiago, H. C., Sands, S. F., Kendrick, D. F., \& Cook, R. G. (1985). Memory processing of serial lists by pigeons, monkeys, and people. Science, 229, 287-289.

\section{NOTES}

1. The question of whether to use conditionalized or unconditionalized results is a difficult one. When initially analyzing single versus double recall trials, we chose to use unconditionalized data because of trial selection concerns. Trials yielding a correct initial recall might simply be trials on which the participant encoded the list well; this creates an unfair comparison with the single recall condition. When comparing the recall effects within a trial, however, we felt that conditionalizing was appropriate. In all conditionalized analyses, we only included participants who provided at least one observation for each trial condition.

2. It is worth noting that most demonstrations of retrieval-induced forgetting use related word lists (e.g., members of the same category). Although items in the present experiments share the property of occurring on the same list, and participants must discriminate among items that occur at temporally related serial positions, it is possible that significantly more interference would be found if list items were related along additional dimensions (e.g., semantic or phonological).

3. Because of conditionalizing, slightly different numbers of trials entered into the calculations for "forward" and "backward" trials: Of the possible forward trials, roughly $83 \%$ were included in the analyses shown in Table 1, in comparison with approximately $90 \%$ of the backward trials. Similar differences apply to the content analyses of Experiments 1 and 2 . However, it is unlikely that these differences played much of a role, especially given that the same patterns were found for the copy condition, in which all trials were considered.

(Manuscript received September 9, 2005; revision accepted for publication November 27, 2005.) 